\title{
A Novel White Matter Fibre Tracking Algorithm Using Probabilistic Tractography and Average Curves
}

\author{
Nagulan Ratnarajah ${ }^{1}$, Andrew Simmons ${ }^{2,3}$, Oleg Davydov ${ }^{4}$, and Ali Hojjatoleslami ${ }^{1}$ \\ ${ }^{1}$ Medical Image Computing, School of BioSciences, University of Kent, U.K. \\ ${ }^{2}$ Neuroimaging Department, Institute of Psychiatry, Kings College London, U.K. \\ ${ }^{3}$ NIHR Biomedical Research Centre for Mental Health at the South London and Maudsley \\ NHS Foundation Trust and Institute of Psychiatry, King's College London, U.K. \\ ${ }^{4}$ Department of Mathematics and Statistics, University of Strathclyde, Glasgow, U.K.
}

\begin{abstract}
This paper presents a novel white matter fibre tractography approach using average curves of probabilistic fibre tracking measures. We compute "representative" curves from the original probabilistic curve-set using two different averaging methods. These typical curves overcome a number of the limitations of deterministic and probabilistic approaches. They produce strong connections to every anatomically distinct fibre tract from a seed point and also convey important information about the underlying probability distribution. A new clustering algorithm is employed to separate fibres into branches before applying averaging methods. The performance of the technique is verified on a wide range of seed points using a phantom dataset and an in vivo dataset.
\end{abstract}

\section{Introduction}

Fibre tractography using diffusion tensor magnetic resonance imaging (DT-MRI) is a promising method for reconstructing the pathways of white matter fasciculi in the human brain noninvasively. A variety of algorithms have been proposed aiming to generate fibre-tract trajectories [1-5]. Generally these algorithms can be categorised into two main types, deterministic and probabilistic. Deterministic approaches are capable of creating anatomically reliable reconstructions of major white matter tracts. However, they do not correctly deal with branching of white matter tracts as such techniques produce only one path per seed point and there is no measure describing the confidence or uncertainty of the reconstructed trajectories. Probabilistic tractography algorithms have been developed to overcome these shortcomings.

The aim of probabilistic tracking methods is to provide a natural approach for modelling uncertainty and generate multiple curves originating from a seed point. Probabilistic methods have also been developed to resolve fibre crossings at the intravoxel level [6] and these methods allow branching of white matter tracts. However, the deterministic tractography approaches have several advantages over probabilistic tractography for some applications such as neurosurgery. Firstly, visualization of the deterministic streamline trajectories is similar to the expected in vivo white matter fibre tracts, whereas the output of probabilistic methods is a connectivity map, which is not a single well-defined trajectory, but rather a spatial distribution. These connectivity maps contain dense 3D volumes of potential connectivities, which cannot be easily inspected. The determination of a connectivity map is also a time-consuming process and requires 
large amounts of memory. Connectivity maps derived using frequency of connection methods demonstrate high frequency connections close to the seed point and low frequency connections at distance from the seed point. This can lead to difficulty in interpreting tracking results, because the derived connection probabilities are not comparable at different distances from the seed point [7]. The connectivity maps from probabilistic tractography are no more than an indication of the number of times that a range of trajectories pass through a voxel from the seed point. The aim of any tractography algorithm is to reconstruct tracts that accurately correlate with the underlying white matter pathways. Secondly, output tracts from probabilistic methods can leak into unexpected regions producing incorrect white matter connections [8].

In this study, we present a novel fibre tractography algorithm using average curves of the output of a probabilistic method, which overcomes a number of the limitations of deterministic and probabilistic tractography techniques described above. The performance of the algorithm has been evaluated using images of a phantom and an in vivo data.

\section{Methodology}

We generate a number of tracts from a seed point based on a probabilistic algorithm; we then divide the curves into two sections, forward and backward from the seed point, then each of the two sets of curves are separated further using a clustering algorithm to find all branch sets. Curves that are very short/long compared to the average arc-length of curves of each branch and curve branches with small number of tracts are deleted from the set. Finally the average curves methods were applied for each set of branched curves from the seed point and the resultant curves concatenated. The tractography algorithm connecting the above steps is described in Algorithm 1. Figure 1 illustrates the concept of our method with the example of a seed point from the phantom data using random walk curves and mean averaging.

We investigated the use of three probabilistic algorithms (a wild bootstrapping [5], a Bayesian [4] and a Random walk [3]) in this work. Two pair-wise distances between curves $\gamma_{\mathrm{i}}$ and $\gamma_{\mathrm{j}}$ were implemented for clustering and median averaging algorithms:

$$
\begin{aligned}
& \text { 1. The Hausdorff distance: } \mathrm{H}\left(\gamma_{\mathrm{i}}, \gamma_{\mathrm{j}}\right)=\max \left[\mathrm{H}^{\prime}\left(\gamma_{\mathrm{i}}, \gamma_{\mathrm{j}}\right), \mathrm{H}^{\prime}\left(\gamma_{\mathrm{j}}, \gamma_{\mathrm{i}}\right)\right] \text {, } \\
& \text { where } H^{\prime}\left(\gamma_{\mathrm{i}}, \gamma_{\mathrm{j}}\right)=\max _{x \in \gamma_{\mathrm{i}}} \min _{y \in \gamma_{\mathrm{j}}}\|x-y\| \text {, }
\end{aligned}
$$

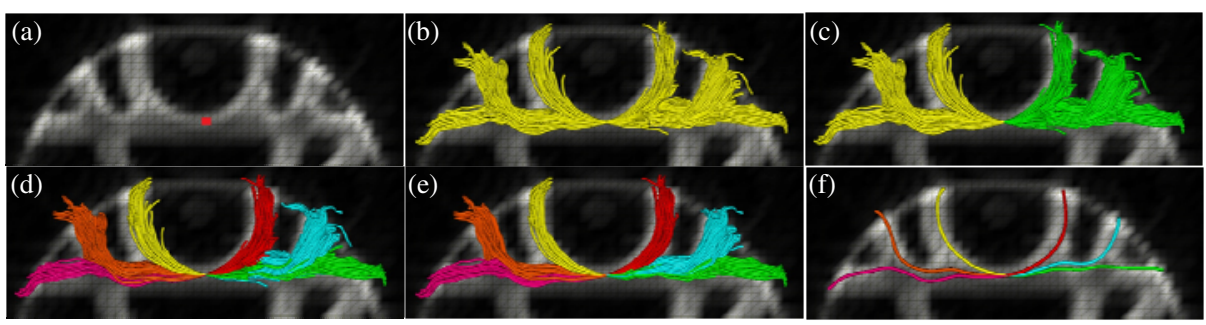

Fig. 1. Overview of the framework of our tractography method (a) Seed point, (b) probabilistic tracking from the seed point, (c) forward and backward tracks, (d) clustered branches, (e) branches after pre-processing and (f) average curves 
2. The average closest distance: $G\left(\gamma_{i}, \gamma_{j}\right)=\operatorname{mean}\left(G^{\prime}\left(\gamma_{i}, \gamma_{j}\right), G^{\prime}\left(\gamma_{j}, \gamma_{i}\right)\right)$,

$$
\text { where } G^{\prime}\left(\gamma_{i}, \gamma_{j}\right)=\operatorname{mean}_{x \in \gamma_{i}} \min _{y \in \gamma_{j}}\|x-y\|
$$

Here, we define the mathematical framework for representing and averaging probabilistic fibre tracking curves in $\mathbb{R}^{3}$. Let $\Gamma$ be a set of $N$ probabilistic fibre tracking curves from a seed point, where each curve $\gamma_{\mathrm{i}}$ is defined by a set of $k_{i}$ ordered points in $m=3$ dimensions, represented by a $k_{i} \times m$ matrix.

$\Gamma=\left\{\gamma_{\mathrm{i}}, \quad 1 \leq \mathrm{i} \geq \mathrm{N}, \gamma_{\mathrm{i}} \in \mathcal{M}_{\mathrm{k}_{\mathrm{i}}, \mathrm{m}}\right\}$, Here $\mathcal{M}_{\mathrm{n}, \mathrm{m}}$ denotes the set of all $\mathrm{n} \times \mathrm{m}$ matrices.

\subsection{Clustering}

We developed a distance based divisive hierarchical clustering scheme that uses various curve distance metrics to find all branches. The fibre tracking algorithm and the

\section{Algorithm 1. The tractography algorithm.}

Given a DTI volume, a probabilistic method and predefined parameters: $l$ for branching and $\mathrm{r}, \mathrm{t}, \mathrm{c}$ for pre-processing.

1. Using a given probabilistic tracking method, generate $\mathrm{N}$ tracts as a set of curves $\Gamma$ from a seed point.

2. Divide the curves $\Gamma$ based on their direction forward and backward from the seed point.

$$
\begin{aligned}
& \Gamma_{1}=\left\{\gamma_{\mathrm{i}}^{1}, 1 \leq \mathrm{i} \geq \mathrm{N}, \gamma_{\mathrm{i}}^{1} \in \mathcal{M}_{\mathrm{k}_{\mathrm{i}}^{1}, \mathrm{~m}}\right\} \text { and } \Gamma_{2}=\left\{\gamma_{\mathrm{i}}^{2}, 1 \leq \mathrm{i} \geq \mathrm{N}, \gamma_{\mathrm{i}}^{2} \in \mathcal{M}_{\mathrm{k}_{\mathrm{i}}^{2}, \mathrm{~m}}\right\}, \\
& \text { where } \mathrm{k}_{\mathrm{i}}=\mathrm{k}_{\mathrm{i}}^{1}+\mathrm{k}_{\mathrm{i}}^{2}-1
\end{aligned}
$$

3. Define the branch sets $\Gamma_{1, \mathrm{p}}, \mathrm{p}=1 \ldots \mathrm{P}$ and $\Gamma_{2, \mathrm{q}}, \mathrm{q}=1 \ldots \mathrm{Q}$ for $\Gamma_{1}$ and $\Gamma_{2}$, using the clustering algorithm (threshold $l$ ), where $\mathrm{P}$ and $\mathrm{Q}$ are the number of branches.

$$
\begin{aligned}
& \Gamma_{1, \mathrm{p}}=\left\{\gamma_{\mathrm{i}}^{1, \mathrm{p}}, 1 \leq \mathrm{i} \geq \mathrm{N}_{1, \mathrm{p}} \gamma_{\mathrm{i}}^{1, \mathrm{p}} \in \mathcal{M}_{\mathrm{k}_{\mathrm{i}}^{1, \mathrm{p}}, \mathrm{m}}\right\}, \sum \mathrm{N}_{1, \mathrm{p}}=\mathrm{N} \text { and } \\
& \Gamma_{2, \mathrm{q}}=\left\{\gamma_{\mathrm{i}}^{2, \mathrm{q}}, 1 \leq \mathrm{i} \geq \mathrm{N}_{2, \mathrm{q}} \gamma_{\mathrm{i}}^{2, \mathrm{q}} \in \mathcal{M}_{\mathrm{k}_{\mathrm{i}}^{2, \mathrm{q}}, \mathrm{m}}\right\}, \sum \mathrm{N}_{2, \mathrm{q}}=\mathrm{N}
\end{aligned}
$$

4. Pre-processing

a. Delete branch set if number of curves in branch $<r \%$ of $N$

b. Delete curves in every branch set if the arc-length of the curve $<\mathrm{t} \%$ or $>\mathrm{c} \%$ of the average arc-length of the curves in the branch

c. Output: $\Gamma_{\mathrm{b}}=\left\{\gamma_{\mathrm{i}}^{\mathrm{b}}, 1 \leq \mathrm{i} \geq \mathrm{N}_{\mathrm{b}}, \gamma_{\mathrm{i}}^{\mathrm{b}} \in \mathcal{M}_{\mathrm{k}_{\mathrm{i}}^{\mathrm{b}}, \mathrm{m}}\right\}$,

$$
\mathrm{b}=1, \ldots, \mathrm{B} \text {, where } \sum \mathrm{N}_{\mathrm{b}} \leq \mathrm{N} \text { and } \mathrm{B} \leq \mathrm{P}+\mathrm{Q}
$$

5. Estimate average curve $\gamma_{\mathrm{avg}}^{\mathrm{b}}$ for each branch set $\Gamma_{\mathrm{b}}$ and concatenate each resulting average curve $\gamma_{\mathrm{avg}}^{\mathrm{b}}$ giving output $\gamma_{\mathrm{s}}$ from seed point $\mathrm{s}$.

6. Repeat from step 1 for a new seed point. 
separation at the seed point provides a set $\Gamma_{k}, k=1$ or 2 of $3 \mathrm{D}$ curves $\gamma_{\mathrm{i}}$. Given a pair-wise distance $d$ and a fibre $\gamma_{i}, d$ is computed between $\gamma_{i}$ and $\gamma_{j}$ for all $\gamma_{j}$ in $\Gamma_{k}$, $\mathrm{j} \neq \mathrm{i}$. A similarity matrix $D$ is defined which organizes the pair-wise distances $\mathrm{d}$ between each pair of curves.-Matrix $D$ is then used in a classical divisive hierarchical clustering algorithm, described by the following steps.

1) Clustering algorithms begins with all the curves as a single cluster.

2) Select the maximum value in the $D$ and find the corresponding curves $\gamma_{\mathrm{C} 1}$ and $\gamma_{\mathrm{C} 2}$.

3) Divide the curves $\gamma_{i}, i \neq c 1, c 2$, into two clusters, one related to $\gamma_{\mathrm{C} 1}$ and the other related to $\gamma_{\mathrm{C} 2}$ using a measure $\operatorname{Min}\left(\mathrm{d}\left(\gamma_{\mathrm{i}}, \gamma_{\mathrm{C} 1}\right), \mathrm{d}\left(\gamma_{\mathrm{i}}, \gamma_{\mathrm{C} 2}\right)\right)$.

Repeat steps 2 and 3 for every cluster and successively divide the curves into a hierarchy of smaller and smaller clusters until the maximum value $<l$, where $l$ is a threshold to be chosen.

\subsection{Average Curves}

We consider a representative curve from a given collection of curves in space as the average curve of the collection. Clearly the representative curve needs to be as close as possible to all curves in the collection. This can be achieved by ensuring that the average curve is that which minimises the difference from all the other curves. Two types of average measures are implemented for this work.

Mean Curve: The mean curve is calculated using an arc-length re-parameterisation method, which re-parameterises the curves by placing a high number of points at equal arc-length steps on each curve. In our implementation, we used a parameter for deciding the constant arc-length step based on the average arc-length of the set of curves in the branch to reparameterize the curves. We consider the collection of parametric curves as being an independent realization of a probabilistic process $\boldsymbol{\Gamma}(\mathbf{t})$ that has mean $\mathbf{E}\{\boldsymbol{\Gamma}(\mathbf{t})\}=\boldsymbol{\mu}(\mathbf{t})$. A random curve from the population may then be expressed as

$$
\Gamma(\mathrm{t})=\mu(\mathrm{t})+\varepsilon(\mathrm{t})
$$

where $\varepsilon(\mathrm{t})$ are independent and $E(\varepsilon(t))=0$. For non parametrical estimation of the overall mean function $\mu(t)$, we use the least squares estimate of $\mu$, which is obtained by averaging the data values separately at each parameter value $t$.

$$
\hat{\mu}(t)=\frac{1}{p(t)} \sum_{i=1}^{p(t)} x_{i}(t), \quad i=1,2, \ldots \operatorname{Max}
$$

where $\mu \in \mathcal{M}_{\text {Max,m }}, \mathrm{p}(\mathrm{t})$ is the number of curves involved in the calculation and Max is the maximum number of points placed in the reparameterization process.

Median Curve: The median curve is selected from the collection as the curve which differs least from all other curves. This is computationally easier than constructing a new curve. We use the matrix of pair-wise distances $\mathrm{D}$, described in section 2.1 , to identify the best curve(s) from the set of likely curves in the branch-set. We use a hierarchical algorithm to find the median curve with the following steps. 
1) Select the maximum value in the $D$ and find the correspondent curves $\gamma_{C 1}$ and $\gamma_{C 2}$.

2) Remove the two curves $\gamma_{\mathrm{C} 1}$ and $\gamma_{\mathrm{C} 2}$ from the set.

3) Repeat steps 1 and 2 for every remaining curve-set and successively remove the curves into a hierarchy of smaller and smaller numbers of curves until one or two curves remain in the set.

If the number of resultant curves is two, the mean curve approach described above is applied to the resultant curves to produce a single curve.

\subsection{Data Acquisition}

Diffusion-weighted data were acquired from a physical phantom [9] on a 3T MRI system with $3 \times 3 \times 3 \mathrm{~mm}^{3}$ voxel resolution, $\mathrm{b}$ value $=1500 \mathrm{~s} / \mathrm{mm}^{2}$ and 64 diffusion directions. We applied the average curves algorithm to the phantom data, using three probabilistic tracking methods, described above, from 16 pre-defined seed positions with 1000 iterations. The same fibre structures were then extracted using the FACT [2] deterministic algorithm using the same parameters.

Quantitative Error Analysis: The ground truth curves $\gamma_{\mathrm{T}}$ is the ideal trajectory and $\gamma_{\mathrm{s}}$ the resultant curves using the average curves method of most probable branches and FACT method from the 16 seed points of the phantom data. The two most probable branches from the forward and backward curve sets are selected from the set of different branches from the seed point as that containing the highest number of curves. Performance measures $(\xi)$ were calculated using the average closest distance $\mathrm{G}^{\prime}$ from $\gamma_{\mathrm{S}}$ to $\gamma_{\mathrm{T}}$, and the Hausdorff distance $\mathrm{H}^{\prime}$ from $\gamma_{\mathrm{S}}$ to $\gamma_{\mathrm{T}}$.

Diffusion weighted images were acquired from a healthy human on a 1.5 Tesla scanner with 64 diffusion encoding gradients and a b-value of $1000 \mathrm{~s} / \mathrm{mm}^{2}(2 \times 2 \times 2$ $\mathrm{mm}^{3}$ voxels). Random trajectories were initiated from a seed point in the corpus callosum and two points in the right/left internal capsule using Bayesian and wild bootstrapping probabilistic methods respectively.

\section{Results}

Figure 2 shows the typical results of average curves of the most probable branches for each seed point of the phantom data. The average curve results are consistent with the ground truth except where some paths meet crossing regions. A comparison of our method with an implementation of the FACT deterministic method using the same seed points showed that our algorithm is more robust in the presence of complex pathways. The FACT trajectories show unusual tract behaviour, sharp bends and loops, while the average curve trajectories generally did not. The lengths of the resulting FACT tracking curves were also smaller than the average curve lengths.

Table 1 presents mean and standard deviation values for the performance measures $(\xi)$ for the 16 pre-defined seed points of the phantom data. The average curve methods are more accurate than the streamline tracking methods with significantly lower errors. The mean curve results are considerably lower than the results of the two distance median curves. Here we used asymmetric measures to estimate the error, in order to avoid accumulation of errors due to early stopping curves or mis-directed paths. 
Table 1. The performance measures $(\xi)$ in $\mathrm{mm}$ (F -FACT method, D-Distance, A-Average closest distance, H-Hausdorff distance, W-Wild bootstrap, B-Bayesian, R-Random Walk).

\begin{tabular}{|c|c|c|r|r|r|r|r|r|r|r|}
\hline \multirow{2}{*}{ D } & \multirow{2}{*}{ F } & \multicolumn{3}{|c|}{ Mean curve } & \multicolumn{3}{c|}{ Median Curve(A) } & \multicolumn{3}{|c|}{ Median Curve(H) } \\
\cline { 3 - 11 } & & W & B & R & W & B & R & W & B & R \\
\hline \multirow{3}{*}{$\mathbf{A}$} & 6.25 & 2.82 & 2.95 & 3.18 & 3.08 & 3.31 & 3.67 & 3.51 & 3.96 & 4.08 \\
& \pm 7.6 & \pm 1.3 & \pm 1.6 & \pm 1.9 & \pm 0.8 & \pm 1.6 & \pm 2.1 & \pm 2.1 & \pm 1.6 & \pm 2.9 \\
\hline \multirow{2}{*}{ H } & 17.6 & 8.75 & 9.74 & 11.07 & 8.73 & 11.04 & 13.04 & 11.07 & 11.08 & 14.63 \\
& \pm 27.8 & \pm 8.3 & \pm 8.2 & \pm 10.1 & \pm 7.6 & \pm 10.2 & \pm 11.4 & \pm 11.1 & \pm 11.1 & \pm 14.1 \\
\hline
\end{tabular}

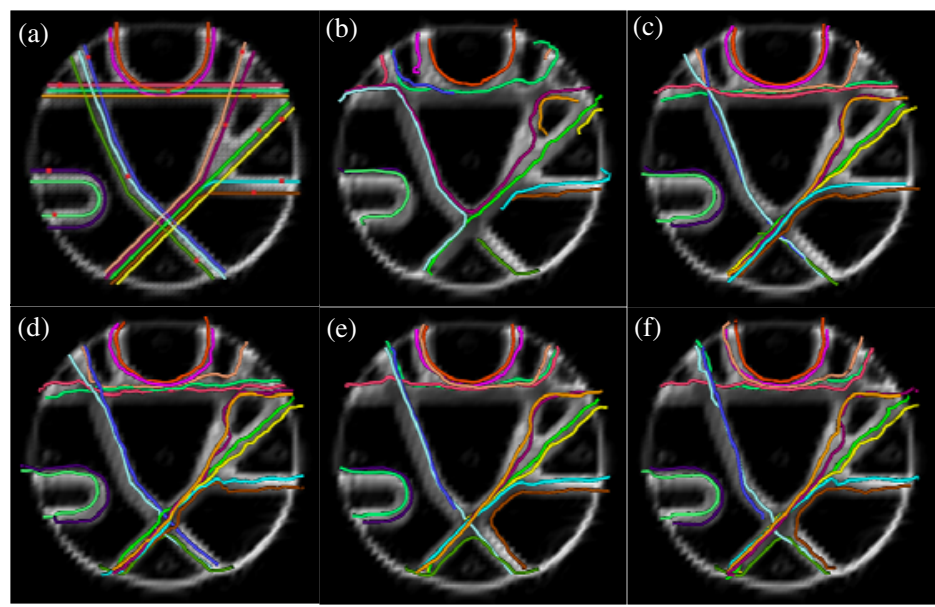

Fig. 2. (a) Ground truth and seed points and results of (b) FACT (c) mean and (d) median curves (A) of wild bootstrapping and (e) mean and (f) median curves (A) of Bayesian tracking

The desired results observed from performing clustering of the corpus callosum (Figure 3) are its division into anatomic regions according to the fibre projections. Mean and median curves of the clusters show representative connections from the seed point to different regions. Using more than one seed point will increase the number of branches and average curves. However, we have illustrated the results using a single seed point in order to provide a clear view of the average curves and to simplify the evaluation.
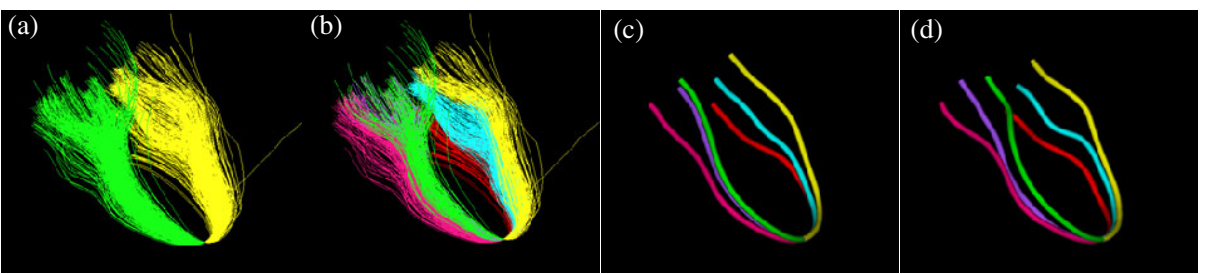

Fig. 3. Tractography results using Bayesian probabilistic tracking from a seed point in the corpus callosum (a) Forward and backward tracts (b) clustered tracts (c) mean curves (d) median curves $(\mathrm{A})$ 


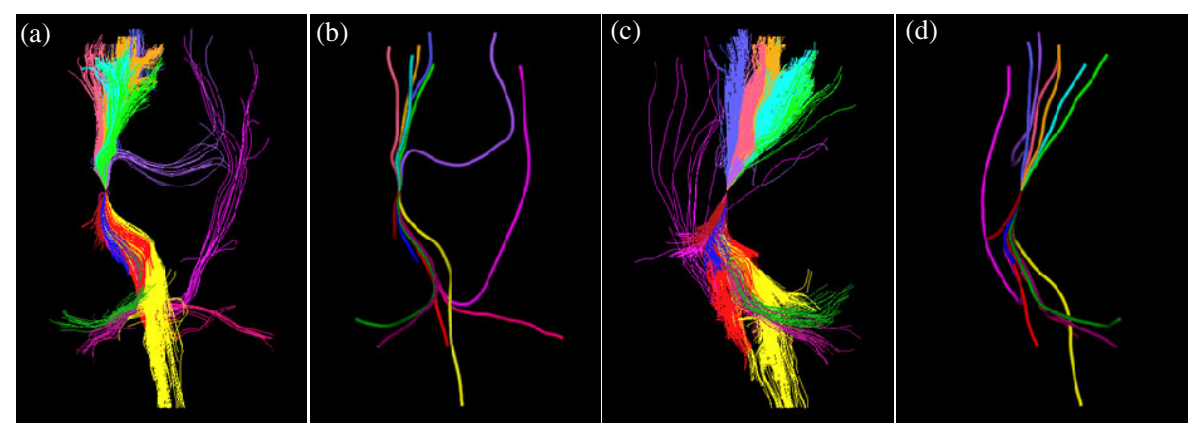

Fig. 4. Tractography results using wild bootstrap tracking obtained from a seed point placed in the cortico-spinal tract. (a), (c) Clustered trajectories and (b), (d) mean curves. (a), (b) front view and (c), (d) lateral view.
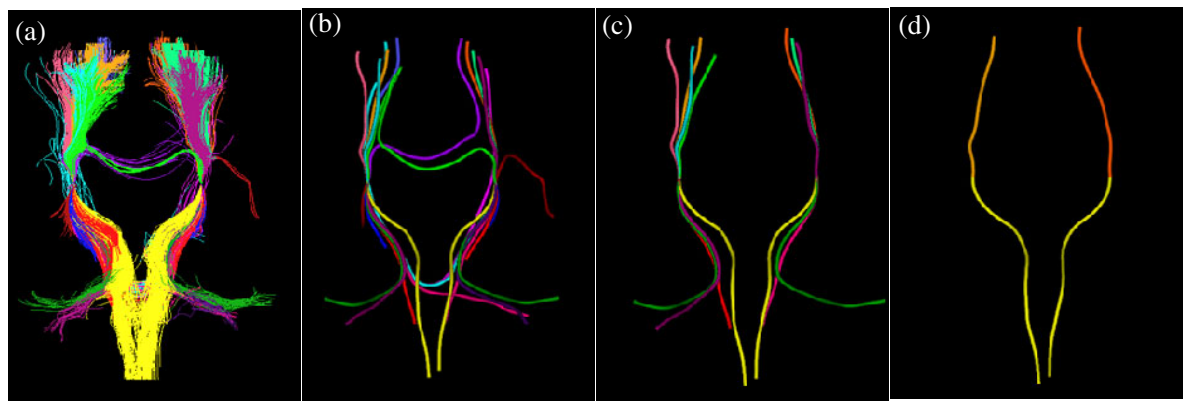

Fig. 5. Tractography results using wild bootstrap tracking obtained from a seed point placed in the left/right cortico-spinal tract from a front view. (a) Clustered trajectories, (b) mean curves, (c) mean curves of branches which contain more than $20 \%$ of the total number of generated curves and (d) mean curve of most probable branches.

The estimated corticospinal tracts (Figure 4 and 5) propagate inferiorly through the internal capsule and the results show that our average curves properly reconstruct the fibres to the different motor areas. The clustering result shows that the tracts were grouped into different plausible bundles. However, the outputs of the wild bootstrapping method showed that a number of deterministic curves erroneously cross the pons and project into the contralateral hemisphere. Figures 5 (c) and (d) show that anatomically implausible pathways are mostly represented by branches with low number of curves. When thresholded at $>20 \%$ number of curves, the results demonstrate plausible routes.

\section{Discussion}

In this paper, we have introduced a novel fibre tractography method based on average curves of probabilistic methods, which results in a single well-defined trajectory for every strong anatomically distinct connection from a seed point by combining the 
advantages of both deterministic and probabilistic algorithms. We evaluated the average curves algorithm on both phantom and in vivo data. The overall shape of the fibre tract trajectories has been shown to correspond with known anatomy, providing quantitatively useful data. However, there are conceptual and practical issues that must be understood when choosing this approach. The results of our average curves approach depend on the probabilistic methods which we applied. While the probabilistic methods still suffer from the general problems of tractography, relying only on the overall shape of trajectories gives an inherent degree of protection against the effects of noise and partial volume.

The accuracy of fibre tractography is influenced by the diffusion tensor measurements' sensitivity to image noise and various other factors. Several studies have investigated the accuracy of deterministic tractography algorithms. We have used average curves as a tool to analyse errors in these probabilistic methods.

The results of our tractography algorithm show that the method handles branching correctly and addresses many of the difficulties faced by traditional probabilistic methods. The average curves were also shown to be good representations of optimal fibre paths of strong connections using both phantom and in vivo data. We have also presented new techniques for clustering probabilistic curves in $3 \mathrm{D}$, to find anatomically distinct branches and remove outlier curves. The average curve methods we describe here are fast and relatively easy to implement. One limitation of our study is that we use some semi-automated parameters to identify the short curves and separate the curves from generated curves.

\section{References}

1. Basser, P.J., Pajevic, S., Pierpaoli, C., Duda, J., Aldroubi, A.: In vivo fiber tractography using DT-MRI data. Magn. Reson. Med. 44, 625-632 (2000)

2. Mori, S., Crain, J., Chacko, V.P., Van Zijl, P.C.M.: Three dimensional tracking of axonal projections in the brain by magnetic resonance imaging. An. Ne. 45(2), 265-269 (1999)

3. Hagmann, P., Thiran, J.P., Jonasson, L., Vandergheynst, P., Clarke, S., Maeder, P., Meuli, R.: DTI mapping of human brain connectivity: statistical fibre tracking and virtual dissection. NeuroImage 19(3), 545-554 (2003)

4. Friman, O., Farnback, G., Westin, C.F.: A Bayesian approach for stochastic white matter tractography. IEEE Trans. Med. Imaging 25(8), 965-978 (2006)

5. Jones, D.K.: Tractography gone wild: probabilistic fibre tracking using the wild bootstrap with diffusion tensor MRI. IEEE Trans. Med Imaging 27, 1268-1274 (2008)

6. Behrens, T.E., Woolrich, M.W., Jenkinson, M., Johansen-Berg, H., Nunes, R.G., Clare, S., Matthews, P.M., Brady, J.M., Smith, S.M.: Characterization and propagation of uncertainty in diffusion-weighted MR imaging. Magn. Reson Med. 50(5), 1077-1088 (2003)

7. Morris, D., Embleton, K., Parker, G.: Probabilistic fibre tracking: differentiation of connections from chance events. NeuroImage 42, 1329-1339 (2008)

8. Descoteaux, M., Deriche, R., Lenglet, C.: Diffusion Tensor Sharpening Improves White Matter Tractography. SPIE Image Processing: Medical Imaging, USA (February 2007)

9. Poupon, C., Rieul, B., Kezele, I., Perrin, M., Poupon, F., Mangin, J.F.: New diffusion phantoms dedicated to the study and validation of high-angular-resolution diffusion imaging (HARDI) models. Magnetic Resonance in Medicine 60(6), 1276-1283 (2008) 